\title{
Probabilistic Decentralised Control and Message Passing Framework for Future Grid
}

\author{
Randa Herzallah, Yuyang Zhou
}

\begin{abstract}
In this paper, we propose a unified probabilistic decentralised control and message passing framework for real time control of the electrical grid which enables the development of the future smart grid. The key elements of the proposed framework are the design of local randomised controllers and probabilistic message passing methodology which enables the coordination between the designed local controllers to account for optimisation considerations on the system operation. Within the proposed framework, the electric grid is decomposed into a number of control areas. Additionally, since the frequency is the ubiquitous grid state variable, representing the balancing of active power generation and consumption, the proposed framework is demonstrated based on local Load Frequency Control (LFC). Simulation studies involving a six-area power system and three interconnection schemes are carried out to illustrate the applicability and effectiveness of the proposed approach.
\end{abstract}

Index Terms-Fully Probabilistic Design, Stochastic systems control, Decentralised control, Power systems, Probabilistic message passing

\section{INTRODUCTION}

The current grid can be characterised as one large centrally dispatched supply system cascading hierarchically to meet consumer demand. This hierarchical transmission grid assumes passive consumers, fixed local distribution, fixed microgrids, and has large inertia. Despite being successful, recent development engender a much more dynamic and uncertain grid: intermittent supply of renewables, electrification of heat and transport, deployment of smart meters, interconnectivity of communication systems, a more distributive nature involving active consumers, and particularly very low inertia. Additionally, the legislative and political drivers that the governments need to conform to international agreements, are also driving the future grid to be more volatile, to have the need to deal with uncertainties. This new future grid demands a new control and communications methodology to deal with uncertainty, volatility, robustness and responsiveness. In response to the challenges posed by the most likely future power grid scenario, this paper proposes a fully probabilistic decentralised control and message passing framework to address many of the confounding issues.

The proposed decentralised control framework decomposes the control task of the grid network into smaller tasks where a set of distributed generation units are grouped together to form a mini or microgrid which is then integrated into the power system or operated in an islanded mode. Each mini/micro grid independently manages and controls the power flow in its area and possibly neighboring areas. This partitioning of the grid network and the proposed communication and message passing provide a coordinated system-wide service capability that warrants the effective development of the future smart grid. Since the frequency is the ubiquitous grid state variable, representing the balancing of active power generation and consumption, the proposed framework will be demonstrated based on local Load Frequency Control (LFC) [1]-[3].

Up until now most of the literature on the control of power systems have been limited to deterministic control.

R. Herzallah and Y. Zhou are with the Systems Analytics Research Institute, Aston University, Aston Triangle, Birmingham B4 7ET, UK. (r.herzallah@aston.ac.uk, annamada@163.com)
Specifically, an extended linear quadratic regulator based decentralized algorithm for calculating the optimal control signal for the automatic voltage regulator is developed in [4]. A distributed predictive control architecture that also considers the coordination of the renewable energy systems with the electrical grid and loads is developed in [5]. An introduction on the most challenging LFC problem in multi-area power systems can be found in text books [6], [7] while comprehensive literature surveys on the topic are provided in [8], [9]. However, the increase in integration of renewable and distributed energy generators, the development of consumer participation, new type of demand, as well as frequently changing stakeholder, are driving this ever-growing grid to be more volatile and inherently probabilistic. In this challenging operational environment, it can be difficult to decide on the most appropriate control action to take in order to efficiently manage, optimise and control the grid and at the same time avoid undesired effects due to the existence of stochastic behaviour and uncertain information. To address the volatility nature of the electric grid, recent advances have considered the development of stochastic decentralised control methods [10][13].

Nonetheless, most of the aforementioned methods are based on the minimisation of a predefined expected cost function which assumes certainty equivalence, therefore, it does not generally yield a good performance. Additionally, most of them consider dynamically decoupled subsystems which does not represent the actual architecture of the emerging electrical system. To design reliable and accurate control strategies for the dynamically coupled, stochastic and uncertain electrical systems, we will establish a unified decentralised probabilistic control and message passing framework that is based on the probabilistic state space representation of the electrical areas of the grid network. The proposed framework will be demonstrated on the LFC problem. First, we introduce the key elements and their interactions in the proposed decentralised probabilistic control architecture and discuss the design of the decentralised randomised control strategies which are able to coordinate their actions to account for the overall objective of the electrical grid system. To facilitate the understanding and the analytical solution of the proposed framework we focus on a specific thermal and hydroelectric generation system which is then decomposed into multiple distinct dynamical subsystems, referred to as control areas.

\section{Decentralised Control ArChitecture}

Our proposed decentralised probabilistic control and message passing framework provides an effective way for integrating and coordinating heterogonous distributed generation sources, including for example renewable energy sources, electric vehicles, and combined heat and power systems. What we propose will also be reliable, and designed to deal with and exploit rather than ignore uncertain information. As shown in Figure 1, the proposed decentralised framework considers the decomposition of the electrical grid into $N$ control areas, $\{1,2, \ldots, m, \ldots, N\}$. The key elements of this proposed architecture are the joint design of local randomised controllers, $c\left(u_{i_{k-1}} \mid z_{i_{k-1}}\right), i=1, \ldots N$, and message passing, 
$M_{i \longleftarrow}\left\{x_{j_{k}} \longrightarrow \tilde{x}_{i_{k}}\right\}$ for coordination between the controllers of the individual areas. This decomposition of the grid network may be based on the physical structure of the electrical grid network. Each control area may typically consist of conventional generation sources, renewable energy generation sources and loads. The areas are responsible for meeting the demand of their own consumers and are physically interconnected with each other through transmission lines, referred to as tielines. Physical connections between the different control areas will also be taken into consideration for the purpose of the design and coordination of the decentralised controllers. This allows the mutual transmission and delivery of energy from one control area to another. In particular, when the energy generated in a control area is not enough to satisfy the total power demands in that control area, the additional deficient power may be obtained from neighbouring control areas. On the other hand, when the energy generation in a control area is sufficient and there is a surplus of energy, this area may transmit energy to other neighbouring control areas.

The proposition is to design randomised control strategies for each control area that has the capability to maintain the frequency of each area at its nominal value through local load frequency control. Coordination between the local controllers will be achieved by the use of uncertain information provided as external signals, $\widetilde{x}_{i_{k}}$ from the neighbouring areas. Information diffusion will be achieved through probabilistic message passing, $M_{i \longleftarrow j}\left\{x_{j_{k}} \longrightarrow \tilde{x}_{i_{k}}\right\}$ in order to update the knowledge of the control areas about the states of neighbouring areas which will be achieved by using probabilistic inference methods. Thus, there is also a real-time communication network integrated in the overall system shown as dotted lines in Figure 1. The control areas communicate via the real-time communication network at specific sampling time instants. In Figure 1, the symbols $x_{i_{k}}$ and $u_{i_{k}}$ are used to indicate the state and control input of control area $i$, and the symbol $\tilde{x}_{i_{k}}$ is used to indicate the state of neighbouring areas received by area $i$ through probabilistic message passing. The state of each control area is charachterised by the probability density function (pdf) of the internal state, $s\left(x_{i_{k}} \mid z_{i_{k-1}}\right)$ of that area, and the pdf of the external state, $s\left(\tilde{x}_{i_{k}} \mid \tilde{x}_{i_{k-1}}\right)$ received by that area from neighbouring areas. At sampling instant $k$, control areas broadcast their state information as probabilistic messages to neighbouring areas that they have physical connections with, $M_{i \longleftarrow j}\left\{x_{j_{k}} \longrightarrow \tilde{x}_{i_{k}}\right\}$. Based on the local state information, $x_{i_{k}}$ of the control area and received external state information, $\tilde{x}_{i_{k}}$ from neighbouring areas, the local controller in that control area calculates its own control action. As will become clear from further development, the designed probabilistic controllers are universal controllers which not only estimate the control actions but also the uncertainty around that estimation.

\section{Mathematical model of Multi-Area Power SYSTEM}

Assume that the electrical grid system is decomposed into $N$ control areas which also are sparsely coupled with each other. As discussed earlier, within the proposed decentralised framework, each control area may typically consists of conventional generation sources, renewable energy generation sources and loads. However, to concentrate on the understanding of the proposed probabilistic decentralised control and message passing framework, the power generation in the control areas in this work will be limited to thermal and hydroelectric power stations.

As shown in Figure 2, at steady-state operation, the power sharing through tie-line between two areas, $i$ and $j \in N$, denoted by $P_{t i e, i j}$ is defined as follows,

$$
P_{t i e, i j}=\frac{V_{i} V_{j}}{X_{i j}} \sin \left(\delta_{i}-\delta_{j}\right),
$$

where $X_{i j}$ represents the reactance of the tie-line which connects the two areas $i$ and $j, V_{i}$ and $V_{j}$ stand for the voltages at equivalent terminals of area $i$ and $j$, respectively, and $\delta_{i}, \delta_{j}$ are the power angles of the equivalent machines of area $i$ and $j$, respectively.

For small deviations of the power angles, $\Delta \delta_{i}, \Delta \delta_{j}$ from their equilibrium values, $\delta_{0_{i}}, \delta_{0_{j}}$, the power flow deviation from its nominal value, $\Delta P_{t i e, i j}$ over tie-line $i j$ can be described by the following equation,

$$
\Delta P_{t i e, i j}=T_{i j}\left(\Delta \delta_{i}-\Delta \delta_{j}\right),
$$

where $T_{i j}$ denotes the synchronising torque coefficient and is given by [14], [15],

$$
T_{i j}=\frac{\left|V_{i}\right|\left|V_{j}\right|}{X_{i j}} \cos \left(\delta_{0_{i}}-\delta_{0_{j}}\right) .
$$

Taking the derivative of Equation (2) with respect to time yields,

$$
\Delta \dot{P}_{t i e, i j}=K_{t i e, i j}\left(\Delta f_{i}-\Delta f_{j}\right)
$$

where $K_{t i e, i j}=2 \pi T_{i j}$ represents the synchronisation coefficient between area $i$ and $j$, and $\Delta f_{i}$ and $\Delta f_{j}$ stand for the frequency deviation of each area from their common nominal value, $f_{0}$. Subsequently, the dynamic description of the total power inflow to the $i$-th area from its neighbouring areas $j \in N_{i}$ is given by,

$$
\Delta \dot{P}_{t i e, i}=\sum_{j \in N_{i}} K_{t i e, i j}\left(\Delta f_{i}-\Delta f_{j}\right)
$$

where $\Delta \dot{P}_{t i e, i}$ is the total power inflow to area $i$, and $N_{i}$ represents the set of all neighbouring areas to area $i$.

To obtain a complete mathematical description of the dynamics of area $i$, we consider the model shown in the block diagram depicted in Figure 3 which is widely used in the literature [14]-[16]. From Figure 3, we can see that the total local control signal of the $i$-th area consists of two components; $\Delta P_{C, i}$ which represents the Automatic Generation Control (AGC) signal that needs to be designed, and $\Delta P_{f, i}=-\frac{1}{R_{i}} \Delta f_{i}$ which represents the primary frequency control action. Note that $\Delta P_{f, i}$ is a fixed static control law performed by the speed governor which is a regulating unit attached on the prime mover and will be considered as such in the current paper. Additionally, $R_{i}$ is a static gain referred to as speed droop or speed regulation. Detailed description of this model and speed drrop can be found in [14]-[16].

Based on Equation (4) and Figure 3, the state space representation of each area can be described as follows,

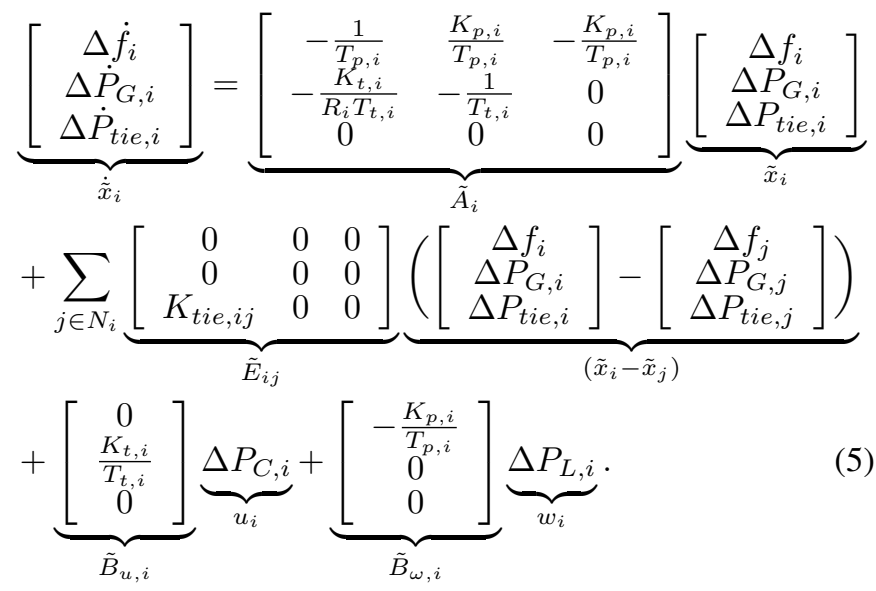

The variable $\Delta P_{G, i}$ in $\tilde{x}_{i}$ is the deviation from the equilibrium value of the electrical power generated by the aggregate 


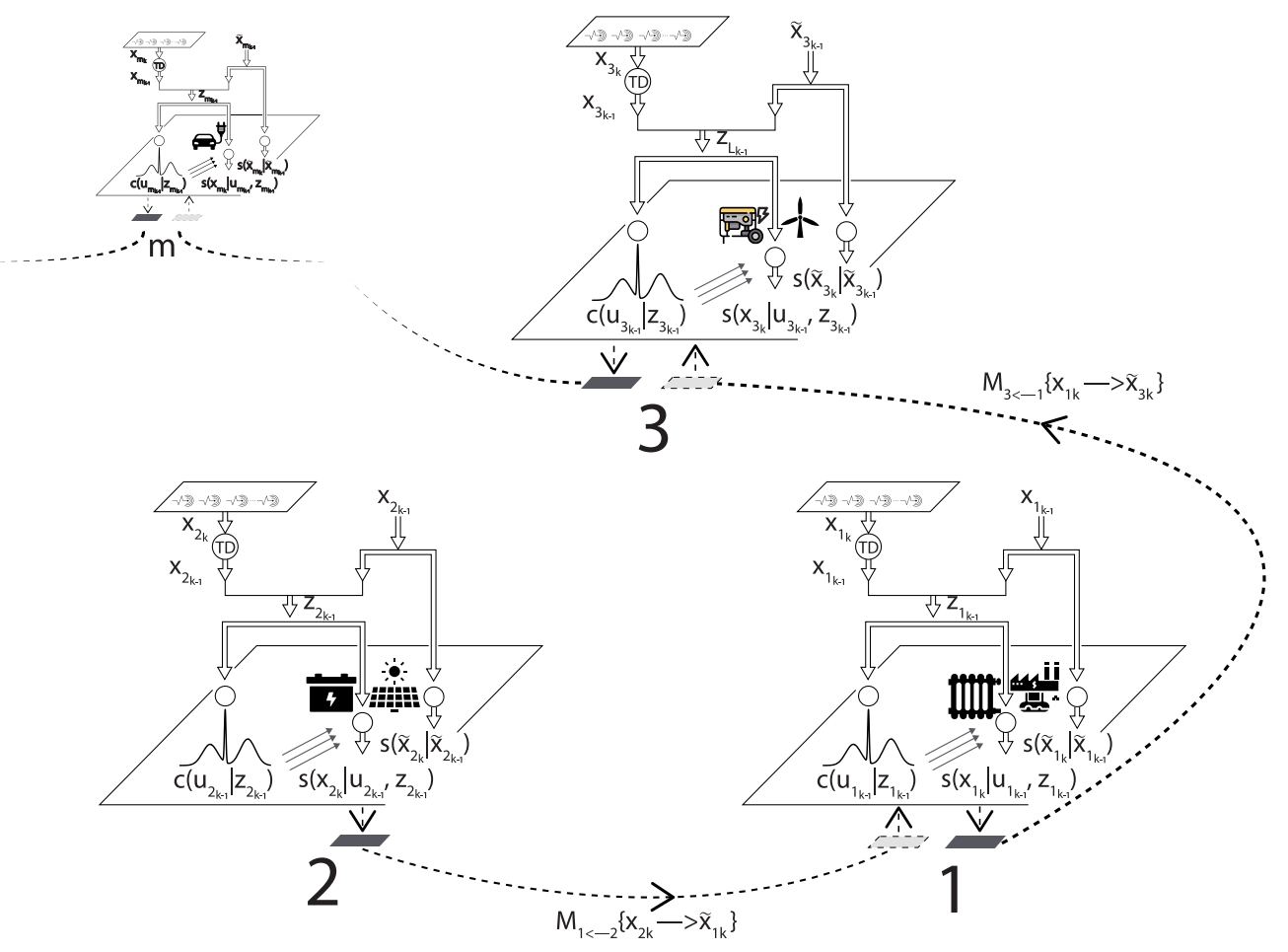

Fig. 1: The architecture of the proposed decentralised control framework and message passing for power systems. The set of sensors in the top parallelogram shape measure the state of the variables, $\tilde{x}_{i_{k}}$ that describe the dynamical behaviour of that area. TD is the temporal delay line.

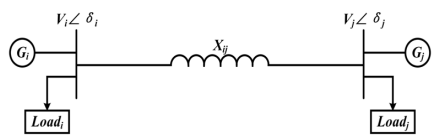

Fig. 2: Tie-line interconnection of two areas system

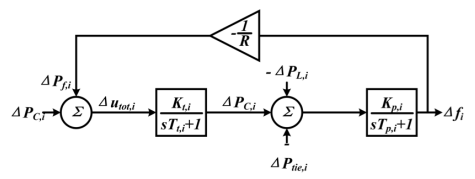

Fig. 3: Single block model of the i-th subsystem.

generating units of each area, and is set to be equal to the mechanical power produced in the output of the turbines. In addition, the disturbance signal $\Delta P_{L, i}$ represents the $i$-th area consumers time-varying demand which is assumed to be unknown, piece wise constant load deviation with known upper and lower bounds, $\Delta P_{L, i, \min }$ and $\Delta P_{L, i, \max }$ respectively.

In order to guarantee zero steady-state error despite the presence of step-disturbances, the system state given in Equation (5) needs to be augmented with an integrator state to introduce integral action [14]. This is achieved by considering a performance variable $y_{i}$ of the $i$-th area as follows,

$$
y_{i}=\beta_{i} \Delta f_{i}+\Delta P_{t i e, i},
$$

where $\beta_{i}$ is a bias factor which is usually taken to be equal to $D_{i}+\frac{1}{R_{i}}$ with $D_{i}$ being the load dependency factor and $R_{i}$ being the speed droop [14], [15]. According to Equation (6), we can see that $y_{i}$, which is also referred to as area control error (ACE) [14], is the summation of the frequency deviation $\Delta f_{i}$ multiplied by $\beta_{i}$ and the total tie-line power inflow $\Delta P_{t i e, i}$. Augmenting the state vector defined in Equation (5) with the performance variable $y_{i}$, yields the following statespace representation,

$$
\begin{aligned}
\dot{x}_{i} & =\underbrace{\left[\begin{array}{cc}
\tilde{A}_{i} & 0_{3 \times 1} \\
C_{y, i} & 0
\end{array}\right]}_{\bar{A}_{1, i}} x_{i}+\sum_{j \in N_{i}} \underbrace{\left[\begin{array}{cc}
\tilde{E}_{i j} & 0_{3 \times 1} \\
0_{1 \times 3} & 0
\end{array}\right]}_{\bar{A}_{2, i j}}\left(x_{i}-x_{j}\right) \\
& +\underbrace{\left[\begin{array}{c}
\tilde{B}_{u, i} \\
0
\end{array}\right]}_{\bar{B}_{u, i}} u_{i}+\underbrace{\left[\begin{array}{c}
\tilde{B}_{\omega, i} \\
0
\end{array}\right]}_{\bar{B}_{\omega, i}} \omega_{i},
\end{aligned}
$$

where $\dot{x}_{i}=\left[\begin{array}{cc}\dot{\tilde{x}}_{i}^{T} & y_{i}\end{array}\right]^{T}$ is the augmented state vector. In addition as can be deduced from Equation (6), $C_{y, i}=\left[\begin{array}{lll}\beta_{i} & 0 & 1\end{array}\right]$.

Discretising Equation (7) using uniform sampling with sampling time $h$, gives,

$x_{i_{k}}=A_{1, i} x_{i_{k-1}}+\sum_{j \in N_{i}} A_{2, i j} x_{j_{k-1}}+B_{u, i} u_{i_{k-1}}+B_{\omega, i} \omega_{i_{k-1}}$,

where $A_{1, i}=h \bar{A}_{1, i}+\sum_{j \in N_{i}} h \bar{A}_{2, i j}+I, A_{2, i}=-h \bar{A}_{2, i j}$, $B_{u, i}=h \bar{B}_{u, i}$, and $B_{\omega, i}=h \bar{B}_{\omega, i}$. In addition, since the electrical system is subject to various sources of uncertainties including for example frequency measurement uncertainties [17], renewable generation uncertainties [18], and transmission line noise [19], a noise term is added to the discretised equation (8) to give,

$$
\begin{aligned}
x_{i_{k}} & =A_{1, i} x_{i_{k-1}}+\sum_{j \in N_{i}} A_{2, i j} x_{j_{k-1}}+B_{u, i} u_{i_{k-1}}+B_{\omega, i} \omega_{i_{k-1}} \\
& +v_{i_{k-1}},
\end{aligned}
$$

where $v_{i_{k-1}} \sim N\left(0, Q_{i}\right)$ stands for white Gaussian noise, and $Q_{i}$ represents the covariance. 
Given the augmented state space representation of control area $i$ defined in Equation (9), the objective then is to design randomised local controllers that would lead automatically to zero steady-state frequency and tie-line power inflow deviations provided that these are driven by step disturbances, $\omega_{i_{k}}=\Delta P_{L, i}$. This will be discussed in the next section.

\section{Decentralised Control and Message Passing FRAMEWORK}

As discussed earlier, the key elements of the proposed framework are the design of local controllers and message passing. Local controllers will be designed such that they maintain the frequency and power inflow of their corresponding areas at their nominal values while at the same time coordinate and harmonise their actions with their neighbouring areas in order to ensure that the frequency of the electrical grid is maintained at the nominal value and the demand requirements of their areas are met. The coordination between the local controllers will be achieved through probabilistic message passing where each control area needs to broadcast its state information to its neighbouring areas. This broadcast state enters the receiving area as external signal. In addition to the coordination element between local controllers, the state of neighbouring areas are coupled as can be seen from the second term in Equation (9). Thus, before discussing the proposed decentralised control and message passing framework we rewrite Equation (9) as follows,

$$
\begin{aligned}
x_{i_{k}} & =A_{1, i} x_{i_{k-1}}+A_{3, i} \tilde{x}_{i_{k-1}}+B_{u, i} u_{i_{k-1}}+B_{\omega, i} \omega_{i_{k-1}} \\
& +v_{i_{k-1}}
\end{aligned}
$$

where we have rewritten the coupling term in Equation (9) in compact form to explicitly state that the states from neighbouring control areas $x_{j_{k-1}}$ enter control area $i$ as external states, $\widetilde{x}_{i_{k-1}}$. Specifically, $A_{3, i}$ is a matrix whose elements are $A_{2, i j}$ such that $A_{3, i}=\left[A_{2, i j}\right]_{j \in N_{i}, j \neq i}$ and $\tilde{x}_{i_{k-1}}$ is a transpose vector whose elements are the states of neighbouring control areas such that $\tilde{x}_{i_{k-1}}=\left[x_{j_{k-1}}^{T}\right]_{j \in N_{i}, j \neq i}^{T}$. Each control area will then build and identify its own predictive model to estimate and predict the states of neighbouring areas in case it looses communication with its neighbours. This is given by,

$$
\tilde{x}_{i_{k}}=A_{4, i} \tilde{x}_{i_{k-1}}+\epsilon_{i_{k-1}},
$$

where $\epsilon_{i_{k-1}} \sim N\left(0, \tilde{Q}_{i}\right)$ is a white Gaussian noise with zero mean and $\tilde{Q}_{i}$ covariance. The dependence of the external state, $\tilde{x}_{i_{k}}$ on the previous external state only stems from our assumption that the inherent dynamics of these external variables cannot be influenced by the inputs $u_{i_{k-1}}$, or internal state $x_{i_{k-1}}$ of the $i$-th local area. This is a logical assumption since the states from neighbouring areas enter area $i$ as external signals with the aim of informing area $i$ about their operational states and allowing the local controllers to coordinate and harmonise their actions.

\section{A. Randomised controller design}

As can be seen from Equation (10), the current value of the state of control area $i$ is driven by a noise term, $v_{i_{k-1}}$ which represents the various sources of uncertainties as explained earlier. This means that complete information about the $i-$ th control area state can be only obtained using probability density functions (pdfs). For the system given in Equation (10), the pdf of the state conditioned on the previous input and state values can be specified at each instant as,

$$
s\left(x_{i_{k}} \mid z_{i_{k-1}}, u_{i_{k-1}}\right) \sim N\left(\mu_{i_{k}}, Q_{i}\right),
$$

where,

$$
\mu_{i_{k}}=A_{1, i} x_{i_{k-1}}+A_{3, i} \tilde{x}_{i_{k-1}}+B_{u, i} u_{i_{k-1}}+B_{\omega, i} \omega_{i_{k-1}},
$$

$Q_{i}$ is the covariance of the noise $v_{i_{k-1}}$, and $z_{i_{k}}=\left[x_{i_{k}}^{T}, \tilde{x}_{i_{k}}^{T}\right]^{T}$. Similarly, the pdf of the external state given in Equation (11) can be specified at each instant as,

$$
s\left(\tilde{x}_{i_{k}} \mid \tilde{x}_{i_{k-1}}\right) \sim N\left(\tilde{\mu}_{i_{k}}, \tilde{Q}_{i}\right)
$$

where,

$$
\tilde{\mu}_{i_{k}}=A_{4, i} \tilde{x}_{i_{k-1}}
$$

and $\tilde{Q}_{i}$ is the covariance of the noise $\epsilon_{i_{k-1}}$. Conspicuously, the state of control area $i$ is determined by both Equations (12) and (14).

For the LFC problem considered in this paper, the aim is to design a control strategy that maintains the frequency and power of local area $i$ around their nominal values. Since complete information about the state of local area $i$ can be only obtained using pdfs as specified in Equations (12) and (14), the control law needs to be ideally derived using a probabilistic framework. This can be achieved by defining the performance measure to be optimised as the Kullback-Leibler divergence (KLD) between the actual joint pdf of the closed loop description of the dynamics of area $i$ and a predefined ideal joint pdf,

$$
\mathcal{D}\left(f \| f^{I}\right)=\int f(F) \ln \left(\frac{f(F)}{f^{I}(F)}\right) d F .
$$

where,

$$
f(F)=\prod_{k=1}^{H} s\left(x_{i_{k}}, \tilde{x}_{i_{k}}, u_{i_{k-1}} \mid z_{i_{k-1}}\right),
$$

is the joint distribution of the closed loop description of the dynamics of area $i$. Based on the chain rule for pdfs [20], this joint distribution can be factorised as follows,

$$
\begin{aligned}
& s\left(x_{i_{k}}, \tilde{x}_{i_{k}}, u_{i_{k-1}} \mid z_{i_{k-1}}\right) \\
& =s\left(x_{i_{k}} \mid z_{i_{k-1}}, u_{i_{k-1}}\right) s\left(\tilde{x}_{i_{k}} \mid \tilde{x}_{i_{k-1}}\right) c\left(u_{i_{k-1}} \mid z_{i_{k-1}}\right) .
\end{aligned}
$$

Additionally,

$$
f^{I}(F)=\prod_{k=1}^{H} s^{I}\left(x_{i_{k}}, \tilde{x}_{i_{k}}, u_{i_{k-1}} \mid x_{i_{k-1}}, \tilde{x}_{i_{k-1}}\right),
$$

is the ideal joint distribution, which can be factorised as follows,

$$
\begin{aligned}
& s^{I}\left(x_{i_{k}}, \tilde{x}_{i_{k}}, u_{i_{k-1}} \mid x_{i_{k-1}}, \tilde{x}_{i_{k-1}}\right) \\
& =s^{I}\left(x_{i_{k}} \mid z_{i_{k-1}}, u_{i_{k-1}}\right) s\left(\tilde{x}_{i_{k}} \mid \tilde{x}_{i_{k-1}}\right) c^{I}\left(u_{i_{k-1}} \mid z_{i_{k-1}}\right),
\end{aligned}
$$

and $H$ represnts the control horizon. Also, note that since $\tilde{x}_{i_{k}}$ enters control area $i$ as external signal, its corresponding ideal pdf is taken to be equal to its actual pdf given in Equation (18). Following the same procedure in [21] the minimum cost function resulting from the minimisation of Equation (16) with respect to the randomised controller $c\left(u_{i_{k-1}} \mid z_{i_{k-1}}\right)$ can then be shown to be given by the following recurrence equation,

$$
\begin{aligned}
& -\ln \left(\gamma\left(z_{i_{k-1}}\right)\right)=\min _{c\left(u_{i_{k-1}} \mid z_{i_{k-1}}\right)} \int s\left(x_{i_{k}} \mid u_{i_{k-1}}, z_{i_{k-1}}\right) \\
& \times s\left(\tilde{x}_{i_{k}} \mid \tilde{x}_{i_{k-1}}\right) c\left(u_{i_{k-1}} \mid z_{i_{k-1}}\right) \\
& \times\left[\ln \left(\frac{s\left(x_{i_{k}} \mid u_{i_{k-1}}, z_{i_{k-1}}\right) c\left(u_{i_{k-1}} \mid z_{i_{k-1}}\right)}{s^{I}\left(x_{i_{k}} \mid u_{i_{k-1}}, z_{i_{k-1}}\right) c^{I}\left(u_{i_{k-1}} \mid z_{i_{k-1}}\right)}\right)\right. \\
& \left.-\ln \left(\gamma\left(z_{i_{k}}\right)\right)\right] d\left(x_{i_{k}}, \tilde{x}_{i_{k}}, u_{i_{k-1}}\right)
\end{aligned}
$$


where $-\ln \left(\gamma\left(z_{i_{k-1}}\right)\right)$ is the minimum cost-to-go function. Full derivation of Equation (21) is provided in our previous publication [22].

Thus, the general solution for the optimal randomised controller as can be obtained from the recurrence functional equation (21), is given in the following proposition [22].

Proposition 1. Given the joint distribution of the dynamics of control area $i$ given in Equation (18) and its ideal joint distribution given in Equation (20) the optimal randomised controller that minimises the cost-to-go function (21) is given by,

$$
c^{*}\left(u_{i_{k-1}} \mid z_{i_{k-1}}\right)=\frac{c^{I}\left(u_{i_{k-1}} \mid z_{i_{k-1}}\right) \exp \left[-\beta\left(u_{i_{k-1}}, z_{i_{k-1}}\right)\right]}{\gamma\left(z_{i_{k-1}}\right)},
$$

where,

$$
\begin{aligned}
& \gamma\left(z_{i_{k-1}}\right)=\int c^{I}\left(u_{i_{k-1}} \mid z_{i_{k-1}}\right) \exp \left[-\beta\left(u_{i_{k-1}}, z_{i_{k-1}}\right)\right] d u_{i_{k-1}}, \\
& \beta\left(u_{i_{k-1}}, z_{i_{k-1}}\right)=\int s\left(x_{i_{k}} \mid u_{i_{k-1}}, z_{i_{k-1}}\right) \\
& \times\left[\ln \frac{s\left(x_{i_{k}} \mid u_{i_{k-1}}, z_{i_{k-1}}\right)}{s^{I}\left(x_{i_{k}} \mid u_{i_{k-1}}, z_{i_{k-1}}\right)}-\ln \left(\tilde{\gamma}\left(x_{i_{k}}, \tilde{x}_{i_{k}}\right)\right)\right] d x_{i_{k}}, \\
& \ln \left(\tilde{\gamma}\left(x_{i_{k}}, \tilde{x}_{i_{k}}\right)\right)=\int s\left(\tilde{x}_{i_{k}} \mid \tilde{x}_{i_{k-1}}\right) \ln \gamma\left(z_{i_{k}}\right) d \tilde{x}_{i_{k}} .
\end{aligned}
$$
[23].

Full derivation of Equations (22)-(23) can be found in [21],

It is also worth mentioning that Equations (22)-(23) provide the general solution of the decentralised fully probabilistic control design method, which is not restricted by the pdf of the system's dynamics or its ideal distribution. The pdfs could be any arbitrary pdfs. On the other hand, for the special case of discrete time linear and Gaussian pdfs of the dynamics similar to the one considered in this paper, an analytic solution of the randomised controller has been obtained and demonstrated in the literature. However, for the considered LFC problem in this paper, we are encountered by two important challenges. These are,

1) As can be seen from Equation (13) and the elements of the matrices $A_{1, i}$ and $B_{u, i}$ given earlier in Section III, the pair $\left(A_{1, i}, B_{u, i}\right)$ has an uncontrollable mode at the origin. To address this problem, for the purpose of designing the randomised controller only, the matrices of the conditional mean of the state space model given in Equation (13) are reformulated following the perturbation procedure proposed in [14]. Specifically, given the perturbation matrix $\mathcal{E}$,

$$
\mathcal{E}=\left[\begin{array}{cccc}
0 & 0 & 0 & 0 \\
0 & 0 & 0 & 0 \\
0 & 0 & e * h & 0 \\
0 & 0 & 0 & 0
\end{array}\right]
$$

with $e<0$ and $|e|$ is assumed to be sufficiently small, the matrices of the conditional mean of the state space model given in Equation (13) are reformulated as follows,

$$
\begin{aligned}
\check{A}_{1, i} & =A_{1, i}+\mathcal{E}, \\
\check{A}_{2, i j} & =A_{2, i j}-\frac{1}{\iota} \mathcal{E}, \\
\check{A}_{3, i} & =\left[\check{A}_{2, i j}\right]_{j \in N_{i}, j \neq i}
\end{aligned}
$$

where $\iota=\left[\lambda_{\max }\right\rceil$, and $\lambda_{\max }$ is the maximum eigenvalue of the Laplacian matrix $\mathcal{L}$ that specifies the connections between the individual areas. In addition $[$.$] denotes the ceiling function$ that maps $\lambda_{\max }$ to the least integer, greater than or equal to $\lambda_{\max }$. To reemphasise, this perturbation of the matrices $A_{1, i}$ and $A_{2, i j}$ is required only for the design of the randomised controller due to the existence of the uncontrollable mode at the origin.

2) The mean of the conditional pdf of the state of the control area given in Equation (13) has an extra term (corresponding to the piece-wise constant disturbance that represents the time-varying demand of the consumers of the $i$-th area) as compared to the mean of the standard representation in conventional fully probabilistic design control method, which is given as follows,

$$
\mu_{i_{k}}=A_{1, i} x_{i_{k-1}}+A_{3, i} \tilde{x}_{i_{k-1}}+B_{u, i} u_{i_{k-1}} .
$$

Therefore, due to the presence of this extra piece-wise constant disturbance term in the control area conditional mean equation (13), the standard analytical solution obtained in the literature will not be optimal for the multi-area LFC problem considered in this paper. In fact, as will be seen from further development, the existence of this extra term will result into different forms of the optimal cost-to-go function as well as randomised controller. This will be derived based on (21) and will be specified in the next theorem.

Based on the two remarks given above, the next theorem states the analytical solution of the randomised controller for the considered LFC problem. The analytical solution of the randomised controller will be based on the following definitions of ideal distributions of control area state and controller,

$$
\begin{aligned}
s^{I}\left(x_{i_{k}} \mid u_{i_{k-1}}, z_{i_{k-1}}\right) & \sim N\left(0, R_{i}\right), \\
c^{I}\left(u_{i_{k-1}} \mid z_{i_{k-1}}\right) & \sim N\left(0, \Gamma_{i}\right),
\end{aligned}
$$

where $R_{i}$ represents the covariance of the ideal distribution of the state of the control area, and $\Gamma_{i}$ is the covariance of the ideal distribution of the randomised controller which indicates the admissible range of the optimal control input. This definition of the ideal distributions is driven by the control objective of the LFC problem, which is aiming at maintaining the frequency and power deviations of the individual local areas equal to zero. The ideal pdf of the external state is taken to be equal to its corresponding actual pdf since as we discussed earlier these signals are external signals and should not be controlled or influenced by the local controller.

Theorem 1. The pdf of the optimal randomised controller minimising the cost-to-go function (21) subject to the pdfs describing the dynamics of control area $i$ given by Equations (12) and (14), and ideal distributions of area $i$ dynamics and control input given by Equations (27) and (28) respectively, is given by,

$$
c\left(u_{i_{k-1}} \mid z_{i_{k-1}}\right), \sim N\left(\bar{u}_{i_{k-1}}, \bar{\Gamma}_{i_{k-1}}\right)
$$

where,

$$
\begin{aligned}
\bar{u}_{i_{k-1}} & =-L_{i_{k-1}} z_{i_{k-1}}+d_{i_{k-1}}, \\
\bar{\Gamma}_{i_{k-1}} & =\left(\Gamma_{i}^{-1}+B_{u, i}^{T} M_{i_{k}} B_{u, i}\right)^{-1}, \\
L_{i_{k-1}} & =\bar{\Gamma}_{i_{k-1}}^{-1} B_{u, i}^{T}\left[M_{i_{k}} \check{A}_{1, i} \quad M_{i_{k}} \check{A}_{3, i}+S_{i, 2_{k}} A_{4, i}\right], \\
d_{i_{k-1}} & =-\bar{\Gamma}_{i_{k-1}}^{-1} B_{u, i}^{T}\left(M_{i_{k}} B_{\omega, i} \omega_{i_{k-1}}+0.5 P_{i, 1_{k}}^{T}\right)
\end{aligned}
$$

and where,

$-\ln \left(\gamma\left(z_{i k-1}\right)\right)=0.5\left[z_{i_{k-1}}^{T} S_{i, k-1} z_{i_{k-1}}+P_{i, k-1} z_{i_{k-1}}+q_{i_{k-1}}\right]$, 
is the quadratic cost function with,

$$
\begin{aligned}
& S_{i, 1_{k-1}}=\check{A}_{1, i}^{T} M_{i_{k}} \check{A}_{1, i}-\check{A}_{1, i}^{T} M_{i_{k}} B_{u, i} \bar{\Gamma}_{i_{k-1}} B_{u, i}^{T} M_{i_{k}} \check{A}_{1, i}, \\
& S_{i, 2_{k-1}}=\check{A}_{1, i}^{T} M_{i_{k}} \check{A}_{3, i}+\check{A}_{1, i}^{T} S_{i, 2_{k}} A_{4, i}-\check{A}_{1, i}^{T} M_{i_{k}} B_{u, i} \\
& \times \bar{\Gamma}_{i_{k-1}} B_{u, i}^{T}\left(M_{i_{k}} \check{A}_{3_{i}}+S_{i, 2_{k}} A_{4, i}\right), \\
& S_{i, 3_{k-1}}=\check{A}_{3, i}^{T} M_{i_{k}} \check{A}_{3, i}+A_{4, i}^{T} S_{i, 3_{k}} A_{4, i}+2 A_{4, i}^{T} S_{i, 2_{k}} \check{A}_{3, i} \\
& \text { - }\left(B_{u, i}^{T} M_{i_{k}} \check{A}_{3, i}+B_{u, i}^{T} S_{i, 2_{k}} A_{4, i}\right)^{T} \\
& \times \bar{\Gamma}_{i_{k-1}}\left(B_{u, i}^{T} M_{i_{k}} \check{A}_{3, i}+B_{u, i}^{T} S_{i, 2 k} A_{4, i}\right), \\
& P_{i, 1_{k-1}}=P_{i, 1_{k}} \check{A}_{1, i}+2 \omega_{i_{k-1}}^{T} B_{\omega, i}^{T} M_{i_{k}} \check{A}_{1, i} \\
& -2 \omega_{i_{k-1}}^{T} B_{\omega, i}^{T} M_{i_{k}} B_{u, i} \bar{\Gamma}_{i_{k-1}} B_{u, i}^{T} M_{i_{k}} \check{A}_{1, i} \\
& \text { - } P_{i, 1_{k}} B_{u, i} \bar{\Gamma}_{i_{k-1}} B_{u, i}^{T} M_{i_{k}} \check{A}_{1, i} \text {, } \\
& P_{i, 2_{k-1}}=P_{i, 2_{k}} A_{4, i}+2 \omega_{i_{k-1}}^{T} B_{\omega, i}^{T} M_{i_{k}} \check{A}_{3, i}+P_{i, 1_{k}} \check{A}_{3, i} \\
& -2 \omega_{i_{k-1}}^{T} B_{\omega, i}^{T} M_{i_{k}} B_{u, i} \bar{\Gamma}_{i_{k-1}} \\
& \times\left(B_{u, i}^{T} M_{i_{k}} \check{A}_{3, i}+B_{u, i}^{T} S_{i, 2_{k}} A_{4, i}\right)-P_{i, 1_{k}} B_{u, i} \bar{\Gamma}_{i_{k-1}} \\
& \times\left(B_{u, i}^{T} M_{i_{k}} \check{A}_{3, i}+B_{u, i}^{T} S_{i, 2_{k}} A_{4, i}\right) \\
& +2 \omega_{i_{k-1}}^{T} B_{\omega, i}^{T} S_{i, 2_{k}} A_{4, i}, \\
& q_{i_{k-1}}=-\omega_{i_{k-1}}^{T} B_{\omega, i}^{T} M_{i_{k}} B_{u, i} \bar{\Gamma}_{i_{k-1}} B_{u, i}^{T} M_{i_{k}} \\
& \times B_{\omega, i} \omega_{i_{k-1}}-0.25 P_{i, 1_{k}} B_{u, i} \bar{\Gamma}_{i_{k-1}} \\
& \times B_{u, i}^{T} P_{i, 1_{k}}^{T}-\omega_{i_{k-1}}^{T} B_{\omega, i}^{T} M_{i_{k}} B_{u, i} \bar{\Gamma}_{i_{k-1}} \\
& \times B_{u, i}^{T} P_{i, 1_{k}}^{T}+q_{i_{k}}-\operatorname{tr}\left(\left(Q_{i}^{-1}-R_{i}^{-1}-S_{i, 1_{k}}\right) Q_{i}\right) \\
& +\operatorname{tr}\left(S_{i, 3_{k}} \tilde{Q}_{i}\right)+\omega_{i_{k-1}}^{T} B_{\omega, i}^{T} M_{i_{k}} B_{\omega, i} \omega_{i_{k-1}} \\
& +P_{i, 1_{k}} B_{\omega, i} \omega_{i_{k-1}} \text {, } \\
& M_{i_{k}}=R_{i}^{-1}+S_{i, 1_{k}} .
\end{aligned}
$$

We have also introduced the following partitioning of the matrices $S_{i, k-1}=\left[\begin{array}{ll}S_{i, 1_{k}} & S_{i, 2_{k}} \\ S_{i, 2_{k}}^{T} & S_{i, 3_{k}}\end{array}\right]$, and $P_{i, k}=$ [ $P_{i, 1_{k}} \quad P_{i, 2_{k}}$ ]. In addition, $\bar{u}_{i_{k-1}}$ and $\bar{\Gamma}_{i_{k-1}}$ are the mean and covariance of the optimal randomised controller of control area $i$ respectively, $L_{i_{k-1}}$ is the controller feedback gain, and $d_{i_{k-1}}$ is a linear shift which manifests from the extra piece-wise constant disturbance term in the control area state equation. Furthermore, $S_{i, k-1}$ represents the discrete Riccati equation, $P_{i, k}$ which also manifests from the extra disturbance input, is referred to in this paper as the disturbance compensation equation, and $q_{i_{k-1}}$ is some positive constant that does not depend on the system state.

Proof. The proof of this theorem can be obtained following the same procedure in our previous publications [21].

As can be seen from Equation (30), only the two blocks, $S_{i, 1_{k-1}}$ and $S_{i, 2_{k-1}}$ defined in Equation (32) of the full Riccati matrix $S_{i, k-1}$ need to be solved. The third block, $S_{i, 3_{k-1}}$ of the Riccati equation does not need to be solved. Similarly, only $P_{i, 1_{k-1}}$ specified in Equation (32) needs to be solved. This decreases the computational efforts in obtaining the optimal randomised control law compared to the global solution

Remark 1. To re-emphasise, compared to the standard randomised controller in FPD [21], [24] the optimal cost-togo function of the considered LFC multi-area problem has additional linear in the state term, $P_{i, k-1}$ as can be seen from Equation (31). Additionally, the mean of the derived randomised controller is shifted by $d_{i_{k-1}}$ as can be seen from Equation (30). The manifestation of these terms in the optimal cost-to-go function and randomised controller is the consequence of the extra piece-wise constant disturbance term in the control area state equation (13).
Based on the derived randomised controller (29) and the probabilistic models of the control areas (12) and (14), the details of the message passing methodology and how local controllers harmonise their actions to contribute to the objective of maintaining the frequency of the grid network at its nominal value and meeting demand requirements will be introduced in the next section.

\section{B. Probabilistic message passing}

This subsection briefly discusses the probabilistic message passing methodology. For more details and the general results of the probabilistic message passing methodology, the readers are referred to [24].

As discussed in previous sections, message passing is key to the successful implementation of the proposed decentralised control framework. Messages passed between the control areas keep the local controllers informed about the states of their neighbouring areas, thus allow them to make informed decisions and harmonise their actions in order to maintain the power and frequency deviations of the grid network at zero. To be more specific, consider the complete description of the interacting variables of control area $j, s\left(x_{j_{k}}, \tilde{x}_{j_{k}}, u_{j_{k-1}} \mid z_{j_{k-1}}\right)$. This control area will then send a message to its neighbouring control area $i$ broadcasting the probabilistic description of its own internal state, $x_{j_{k}}$. This probabilistic message from control area $j$ to control area $i$ is obtained as follows,

$$
\begin{aligned}
M_{i \leftarrow j_{k}} & =\int s\left(x_{j_{k}}, \tilde{x}_{j_{k}}, u_{j_{k-1}} \mid z_{j_{k-1}}\right) \mathrm{d} \tilde{x}_{j_{k}} \mathrm{~d} u_{j_{k-1}} \\
& =\int s\left(x_{j_{k}} \mid z_{j_{k-1}}, u_{j_{k-1}}\right) s\left(\tilde{x}_{j_{k}} \mid \tilde{x}_{j_{k-1}}\right) \\
& \times c\left(u_{j_{k-1}} \mid z_{j_{k-1}}\right) \mathrm{d} \tilde{x}_{j_{k}} \mathrm{~d} u_{j_{k-1}} \\
& =N\left(\mu_{i \leftarrow j_{k}}, \Sigma_{i \leftarrow j_{k}}\right),
\end{aligned}
$$

where,

$$
\begin{aligned}
\mu_{i \leftarrow j_{k}} & =A_{1, j} x_{j_{k-1}}+A_{3, j} \tilde{x}_{j_{k-1}}+B_{u, j} \bar{u}_{j_{k-1}}+B_{\omega, j} \omega_{j_{k-1}}, \\
\Sigma_{i \leftarrow j_{k}} & =Q_{j}+B_{u, j} \Gamma_{j_{k-1}} B_{u, j}^{T} .
\end{aligned}
$$

Notably we have integrated over all variables of control area $j$ except its internal state. This information about the internal state of control area $j$ is then passed to control area $i$ and fused with the prior information, that control area $i$ retains, in the form of external signals about the state of control area $j$. To clarify, Equations (33) and (14) are fused using Bayes' rule by multiplying the two together, which gives,

$$
\begin{aligned}
s\left(\tilde{x}_{i ; f_{k}}\right) & =N\left(\mu_{i \leftarrow j_{k}}, \Sigma_{i \leftarrow j_{k}}\right) N\left(\tilde{\mu}_{i_{k}}, \tilde{Q}_{i}\right) \\
& =N\left(\tilde{\mu}_{i ; f_{k}}, \tilde{\Sigma}_{i ; f_{k}}\right),
\end{aligned}
$$

where,

$$
\begin{aligned}
& \tilde{\mu}_{i ; f_{k}}=\tilde{\mu}_{i_{k}}+K_{i_{k}}\left(\mu_{i \leftarrow j_{k}}-\tilde{\mu}_{i_{k}}\right), \\
& \tilde{\Sigma}_{i ; f_{k}}=\tilde{Q}_{i}-K_{i_{k}} \tilde{Q}_{i} .
\end{aligned}
$$

These equations can then be used to update the parameter $A_{4, j}$ in Equation (15) using linear optimisation methods.

\section{IMPLEMENTATION PROCEDURE OF THE PROPOSED FRAMEWORK}

To apply the optimal controller (29) to the considered LFC problem of power system network, the solutions of the Riccati and the disturbance compensation equations need to be evaluated. For large control horizon $H$, the problem can be considered as infinite horizon control problem which yields steady state (SS) solutions of the Riccati and compensation 
equations and a constant feedback gain control matrix. Here we start by finding the SS solution of the first partition of the Riccati equation, $S_{i, 1_{k}}$. One way to achieve this is to reverse the direction of time. Using the definition of $S_{i, 1_{k}}$ given in Equation (32) and reversing the direction of time, $S_{i, 1_{k}}$ can be modified as follows,

$$
\begin{aligned}
S_{i, 1_{k}} & =\check{A}_{1, i}^{T} M_{i_{k-1}} \check{A}_{1, i}-\check{A}_{1, i}^{T} M_{i_{k-1}} B_{u, i}\left(\Gamma^{-1}\right. \\
& \left.+B_{u, i}^{T} M_{i_{k-1}} B_{u, i}\right)^{-1} B_{u, i}^{T} M_{i_{k-1}} \check{A}_{1, i},
\end{aligned}
$$

where,

$$
M_{i_{k-1}}=R_{i}^{-1}+S_{i, 1_{k-1}} .
$$

To obtain the SS solution of Equation (37), we start by initialising $S_{i, 1}$ randomly, then iterating until a SS solution, $S_{i, 1}$ is attained. Using the obtained SS solution, $S_{i, 1}$, the SS solution $M_{i}$ can then be evaluated. Finally, using $M_{i}$ in $S_{i, 2_{k-1}}$, and $P_{i, 1_{k-1}}$ in Equation (32), the same time reversal procedure can be applied to obtain the SS solutions $S_{i, 2}$ and $P_{i, 1}$. Specifically,

$$
\begin{aligned}
S_{i, 2_{k}} & =\check{A}_{1, i}^{T} M_{i} \check{A}_{3, i}+\check{A}_{1, i}^{T} S_{i, 2_{k-1}} A_{4, i}-\check{A}_{1, i}^{T} M_{i} B_{u, i}\left(\Gamma^{-1}\right. \\
& \left.+B_{u, i}^{T} M_{i} B_{u, i}\right)^{-1}\left(B_{u, i}^{T} M_{i} \check{A}_{3_{i}}+B_{u, i}^{T} S_{i, 2_{k-1}} A_{4, i}\right),
\end{aligned}
$$

$$
\begin{aligned}
& P_{i, 1_{k}}=P_{i, 1_{k-1}} \check{A}_{1, i}+2 \omega_{i_{k-1}}^{T} B_{\omega, i}^{T} M_{i} \check{A}_{1, i} \\
& -2 \omega_{i_{k-1}}^{T} B_{\omega, i}^{T} M_{i} B_{u, i}\left(\Gamma^{-1}+B_{u, i}^{T} M_{i} B_{u, i}\right)^{-1} B_{u, i}^{T} M_{i} \check{A}_{1, i} \\
& -P_{i, 1_{k-1}} B_{u, i}\left(\Gamma^{-1}+B_{u, i}^{T} M_{i} B_{u, i}\right)^{-1} B_{u, i}^{T} M_{i} \check{A}_{1, i}
\end{aligned}
$$

The procedure of the implementation of the probabilistic decentralised control and message passing framework to the considered power system network (10) is summarised as pseudocode in Algorithm 1.

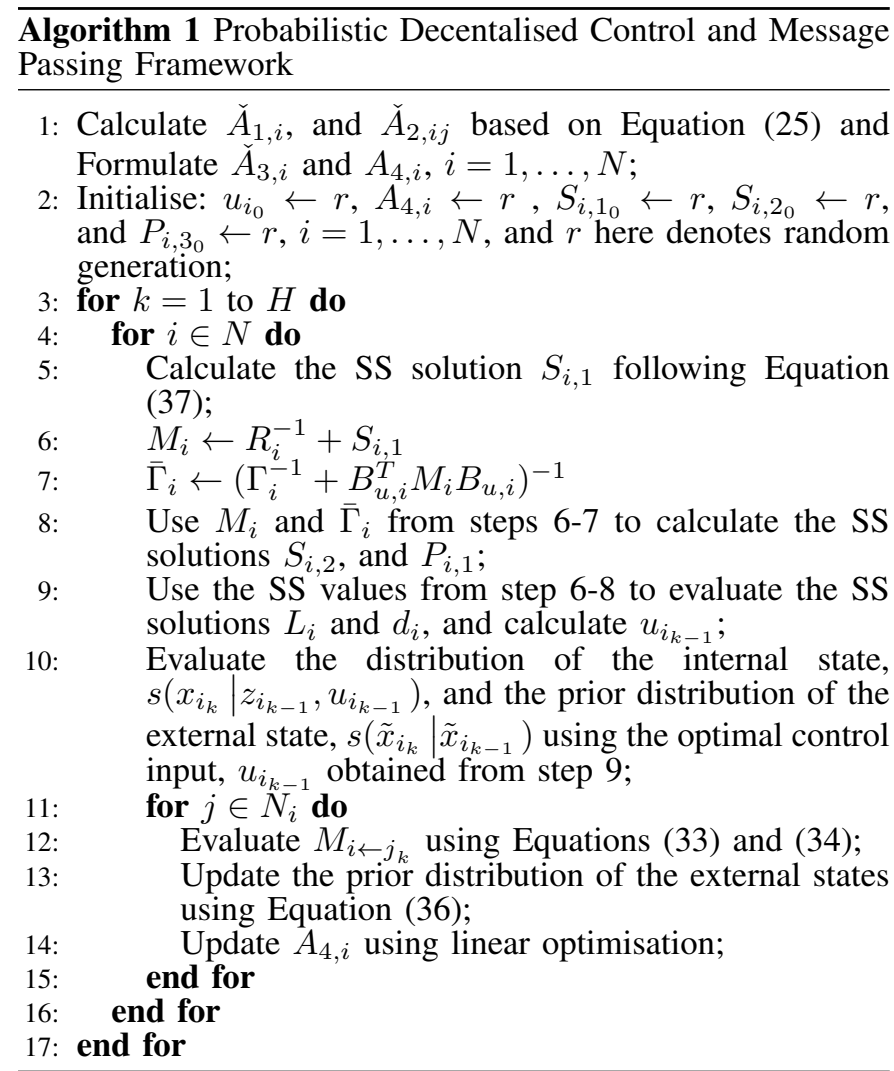

TABLE I: Parameters and power system terminology

\begin{tabular}{ccc}
\hline Symbol and Parameter & Value & Units \\
\hline$f_{0_{i}}$, Nominal Frequency & 50 & $\mathrm{~Hz}$ \\
$P_{B, i}$, Power Base & 2000 & $\mathrm{MW}$ \\
$D_{i}$, Load Dependency Factor & 16.66 & $\mathrm{MW} / \mathrm{Hz}$ \\
$R_{i}$, Speed Droop & $1.2 \times 10^{-3}$ & $\mathrm{~Hz} / \mathrm{MW}$ \\
$H_{i}$, Generator Inertia Gain & 5 & $\mathrm{~s}$ \\
$K_{t, i}$, Turbine Static Gain, & 1 & $\mathrm{MW} / \mathrm{MW}$ \\
$T_{t, i}$, Turbine Time Constant & 0.3 & $\mathrm{~s}$ \\
$K_{p, i}$, Area Static Gain & 0.06 & $\mathrm{~Hz} / \mathrm{MW}$ \\
$T_{p, i}$, Area Time Constant & 24 & $\mathrm{~s}$ \\
$K_{t i e, i}$, Tie-line Coefficient & 1090 & $\mathrm{MW} / \mathrm{Hz}$ \\
\hline
\end{tabular}

\section{Simulation Case Studies}

In this section, the proposed algorithm is tested on three case studies. In these studies, the electrical grid is considered to be decomposed into six control areas, where each case study considers a different topology or interconnection between the control areas. The interconnection schemes of the three considered topologies are shown in Figure 4. As discussed earlier, the individual areas are described mathematically by the model given in Equation (10). The six areas are considered to have identical parameters which are shown in Table I. For the simulation purpose of the three considered cases the sampling time $h$ is taken to be equal to 0.02 . Additionally, the perturbation parameter $e$ is taken to be equal to -0.01 and $\iota=\left[\lambda_{\max }(\mathcal{L})\right]$ is calculated to be equal to 5 . Also, note that external state matrix, $A_{4, i}$ is initialised randomly at the beginning and then updated according to the probabilistic message passing discussed in Section V. Furthermore, in the three case studies the areas are assumed to be subjected to piece-wise constant disturbances which represent the power load variations of the consumers of each area. The times at which these disturbances affect the individual areas and their corresponding values are given in Table II. Finally, the covariance of the external state $\tilde{Q}_{i}$ is assumed to be equal to 0.0005 for all $i$. Besides, in all three cases, the stochastic Gaussian noise $v_{i_{k}}$ of the internal state that describes the dynamic of control area $i$ is assumed to be $v_{i_{k}} \sim N(0,0.001)$. As discussed previously, the local randomised controllers are

TABLE II: Piece-wise Constant disturbances

\begin{tabular}{|c|c|c|}
\hline Control area & Disturbance value & $\begin{array}{c}\text { Effective disturbance } \\
\text { period, sec }\end{array}$ \\
\hline 1 & 200 & $0-140$ \\
2 & -190 & $4-140$ \\
3 & 180 & $9-140$ \\
4 & -170 & $15-140$ \\
5 & 160 & $18-140$ \\
6 & -180 & $25-140$ \\
\hline
\end{tabular}

designed such as to maintain the deviations of the frequency and power in their corresponding areas at zero. Following the procedure stated in Algorithm 1, the frequency and power deviations of the local areas are evaluated for each of the considered topology. The frequency and total power inflow deviation of the first case is shown in Figure 5, (a) and (b). From this Figure, we can see that despite the effect of the noises and power load disturbances, the frequency and total power inflow deviation stay zero. This emphasises that the designed local randomised controllers are able to maintain the frequency and total power inflow at their nominal operating conditions even when their corresponding areas suffer from stochastic disturbances. Similar to case study one, the results of case study two are given in Figure 6, while the results of case study three are provided in Figures 7. From Figures 6-7, we can see that the frequency and total power inflow deviations of all six control areas of case two and case three 
are fluctuating around zero even with the presence of the noise and load step disturbances.

To reemphasise, the randomised controllers are capable of bringing the frequency and total power inflow deviations back to the origin irrespective of the topology, or the effect of noises and disturbances.

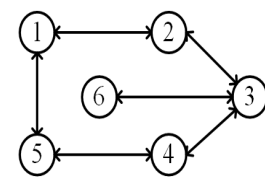

(a)

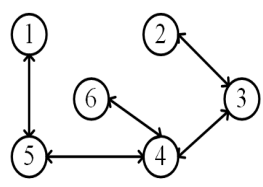

(b)

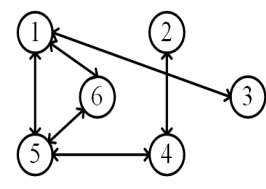

(c)
Fig. 4: Case study 1: (a) $\Delta f_{i}$ for all six areas. (b) $\Delta P_{t i e, i}$ for all six areas.

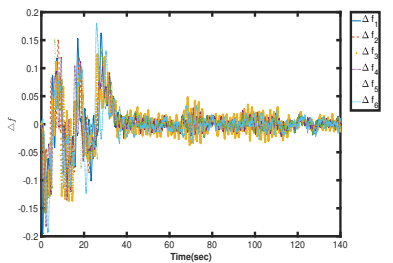

(a)

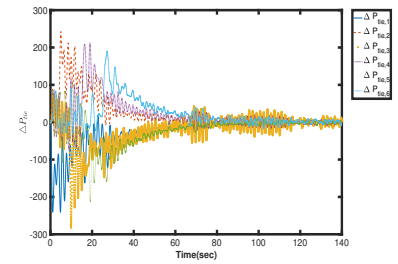

(b)
Fig. 5: Case study 1: (a) $\Delta f_{i}$ for all six areas. (b) $\Delta P_{t i e, i}$ for all six areas.

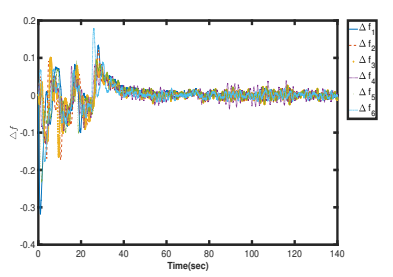

(a)

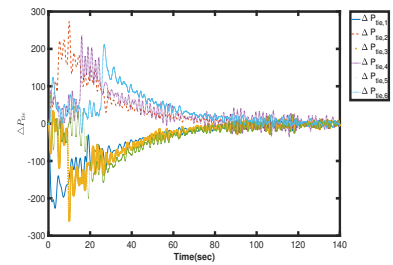

(b)
Fig. 6: Case study 2: (a) $\Delta f_{i}$ for all six areas. (b) $\Delta P_{t i e, i}$ for all six areas.

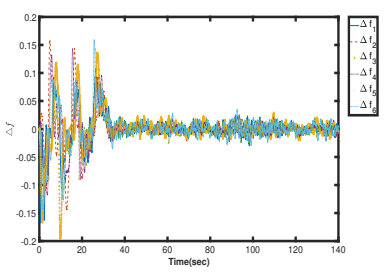

(a)

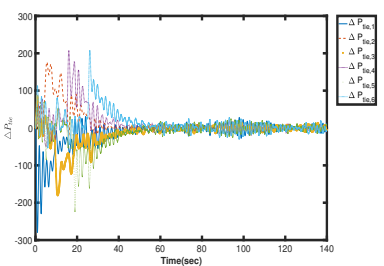

(b)
Fig. 7: Case study 3: (a) $\Delta f_{i}$ for all six areas. (b) $\Delta P_{t i e, i}$ for all six areas.

\section{CONCLUSION}

In response to the urgent and impending need to decentralise the power grid network to accommodate the transition of robust management and control from hundreds to thousands of power generators at present towards tens of millions in the near future, we proposed a unified probabilistic decentralised control and message passing framework. The proposed framework utilises the Kullback-Leibler divergence to derive the optimal local randomised controllers and probabilistic inference for the development of the probabilistic message passing methodology. Within the proposed framework the grid network is decomposed into a number of control areas, and the framework is demonstrated based on local Load Frequency Control (LFC). The proposed framework is shown to be computationally feasible and easily implementable. It also enables the development of the future smart Grid. Simulation studies involving a six-area power system and three interconnection schemes illustrated the applicability and effectiveness of the proposed approach.

\section{REFERENCES}

[1] G. Ray, S. Dey, and T. K. Bhattacharyya, "Multi-area load frequency control of power systems: A decentralized variable structure approach," Electric Power Components and Systems, vol. 33, no. 3, pp. 315-331, 2004.

[2] A. Sina and D. Kaur, "An optimal controller for load frequency control in multi-area deregulated power system," Journal of Electric Systems, vol. 15 , no. 1 , pp. 142-158, 2019

[3] L. Dong, Y. Zhang, and Z. Gao, "A robust decentralized load frequency controller for interconnected power systems," ISA Transactions, vol. 51, no. 3, pp. 410-419, 2012.

[4] K. S. Abhinav and B. C. Pal, "Decentralized control of oscillatory dynamics in power systems using an extended lqr," IIEEE Transactions on Power Systems, vol. 31, no. 3, pp. 1715-1728, 2016.

[5] Q. Wei, L. Jinfeng, and P. D. Christofides, "A distributed control framework for smart grid development: Energy/water system optimal operation and electric grid integration," Journal of Process Control, vol. 21, pp. 1504-1516, 2011.

[6] H. Bevrani, Robust Power System Frequency Control. Berlin/Heidelberg, Germany: Springer, 2010.

[7] J. Ma, Power System Wide-Area Stability Analysis and Control. NJ, USA: Wiley: Hoboken, 2018.

[8] H. Haes Alhelou, M. E. Hamedani-Golshan, R. Zamani, E. HeydarianForushani, and P. Siano, "Challenges and opportunities of load frequency control in conventional, modern and future smart power systems: A comprehensive review," Energies, vol. 21, no. 11, p. 2497, 2018.

[9] S. K. Pandey, S. R. Mohanty, and N. Kishor, "A literature survey on loadfrequency control for conventional and distribution generation power systems," Renewable and Sustainable Energy Reviews, vol. 25, pp. 318 334, 2013.

[10] W. Lin and E. Bitar, "Decentralized stochastic control of distributed energy resources," IEEE Transactions on Power Systems, vol. 33, no. 1, pp. 888-900, 2018.

[11] E. Vrettos, C. Ziras, and G. Andersson, "Fast and reliable primary frequency reserves from refrigerators with decentralized stochastic control," IEEE Transactions on Power Systems, vol. 32, no. 4, pp. 2924-2941, 2017.

[12] P. Ferraro, E. Crisostomi, R. Shorten, and F. Milano, "Stochastic frequency control of grid-connected microgrids," IEEE Transactions on Power Systems, vol. 33, no. 5, pp. 5704-5713, 2018.

[13] R. N. Anderson, A. Boulanger, W. B. Powell, and W. Scott, "Stochastic frequency control of grid-connected microgrids," Proceedings of the $I E E E$, vol. 99, no. 6, pp. 1098-1115, 2011.

[14] E. Vlahakis, L. Dritsas, and G. Halikias, "Distributed lqr design for a class of large-scale multi-area power systems," Energies, vol. 12, no. 14, p. 2664, 2019.

[15] H. Bevrani, Robust power system frequency control. Springer, 2009, vol. 85 .

[16] P. Kundur, N. J. Balu, and M. G. Lauby, Power system stability and control. McGraw-hill New York, 1994, vol. 7.

[17] Y.-H. Moon, H.-S. Ryu, J.-G. Lee, and S. Kim, "Power system load frequency control using noise-tolerable pid feedback," in ISIE 2001. 2001 IEEE International Symposium on Industrial Electronics Proceedings (Cat. No. 01TH8570), vol. 3. IEEE, 2001, pp. 1714-1718.

[18] K. Wang and M. L. Crow, "Numerical simulation of stochastic differential algebraic equations for power system transient stability with random loads," in 2011 IEEE Power and Energy Society General Meeting. IEEE, 2011, pp. 1-8.

[19] K. Chan, A. Chin, C. Kwei, D. Shien, and J. Lin, "Transmission line noise from standard and proton-implanted si," in 2001 IEEE MTTS International Microwave Sympsoium Digest (Cat. No. 01CH37157), vol. 2. IEEE, 2001, pp. 763-766.

[20] V. Peterka, "Bayesian system identification," IFAC Proceedings Volumes, vol. 12, no. 8, pp. 99-114, 1979 .

[21] R. Herzallah and M. Kárnỳ, "Towards probabilistic synchronisation of local controllers," International Journal of Systems Science, vol. 48 no. 3, pp. 604-615, 2017.

[22] R. Herzallah, "A fully probabilistic decentralised control design for complex stochastic systems," in 2019 IEEE 15th International Conference on Control and Automation (ICCA). IEEE, 2019, pp. 806-811.

[23] M. Kárný, "Towards fully probabilistic control design," Automatica vol. 32, no. 12, pp. 1719-1722, 1996.

[24] R. Herzallah, "Probabilistic message passing for decentralized control of stochastic complex systems," IEEE Access, vol. 7, pp. 184 707-184 717, 2019. 